ARTICLE

\title{
Dynamic actin cycling through mitochondrial subpopulations locally regulates the fission-fusion balance within mitochondrial networks
}

\author{
Andrew S. Moore ${ }^{1, \star}$, Yvette C. Wong ${ }^{1, \star}$, Cory L. Simpson ${ }^{1,2} \&$ Erika L.F. Holzbaur ${ }^{1}$
}

Mitochondria form interconnected networks that dynamically remodel in response to cellular needs. Using live-cell imaging, we investigate the role of the actin cytoskeleton in regulating mitochondrial fission and fusion. We identify cycling of actin filaments onto and off of subsets of cellular mitochondria. The association of actin filaments with mitochondrial subpopulations is transient; actin quickly disassembles, then reassembles around a distinct subpopulation, efficiently cycling through all cellular mitochondria within $14 \mathrm{~min}$. The focal assembly of actin induces local, Drp1-dependent fragmentation of the mitochondrial network. On actin disassembly, fragmented mitochondria undergo rapid fusion, leading to regional recovery of the tubular mitochondrial network. Cycling requires dynamic actin polymerization and is blocked by inhibitors of both Arp2/3 and formins. We propose that cyclic assembly of actin onto mitochondria modulates the fission/fusion balance, promotes network remodelling and content mixing, and thus may serve as an essential mechanism regulating mitochondrial network homeostasis.

\footnotetext{
${ }^{1}$ Department of Physiology, Perelman School of Medicine, University of Pennsylvania, 638A Clinical Research Building, 415 Curie Boulevard, Philadelphia, Pennsylvania 19104, USA. ${ }^{2}$ Department of Dermatology, Hospital of the University of Pennsylvania, Philadelphia, Pennsylvania 19104, USA. ${ }^{\star}$ These authors contributed equally to this work. Correspondence and requests for materials should be addressed to E.L.F.H. (email: holzbaur@mail.med.upenn.edu).
} 
$\mathrm{M}$ itochondria are dynamic organelles that undergo fission and fusion to segregate their content of DNA, facilitate transfer of mitochondrial proteins and enable mitophagic clearance of damaged organelles ${ }^{1-3}$. The steady-state balance between mitochondrial fission and fusion is a key determinant of overall mitochondrial network structure and, by extension, cellular bioenergetics ${ }^{4}$. The processes of mitochondrial fission and fusion are regulated by a collection of large GTPases. Mitofusin 1 and Mitofusin 2 control fusion of the mitochondrial outer membrane $e^{5}$, whereas Opal coordinates inner membrane fusion ${ }^{6}$. In contrast, Drp1, through association with specific mitochondria-localized receptors, drives fission of both inner and outer mitochondrial membranes ${ }^{7,8}$.

Before Drp1 recruitment, the endoplasmic reticulum (ER) marks prospective sites of mitochondrial fission ${ }^{9}$. ER tubules twist around mitochondria, inducing a pre-constriction event that decreases the mitochondrial cross-sectional diameter and allows for Drp1 assembly at the site of fission. The force necessary to drive this mitochondrial constriction is provided by actin polymerization by the ER-associated formin INF2 and the mitochondria-anchored formin-activating protein Spire1C $\mathrm{C}^{10-12}$. Consistent with a key role for actin in the initial step of mitochondrial fission, actin depolymerization or depletion of crucial actin-polymerizing proteins results in increased mitochondrial length ${ }^{10,13}$. In addition, actin was shown to directly bind to and activate Drp1, and is robustly recruited to fragmenting mitochondria after treatment with chemical ionophores ${ }^{93,14}$.

Although the contribution of actin to mitochondrial fission has been examined at the level of the individual organelle, much less is known about how the actin cytoskeleton regulates the mitochondrial fission/fusion balance on a cell-wide level. To investigate this question, we used live-cell imaging to examine the dynamic interactions of actin with mitochondria. We made the unexpected observation that filamentous actin (F-actin) cyclically associates with distinct mitochondrial subpopulations in an Arp2/3- and formin-dependent manner, to regulate mitochondrial networks within the cell. Actin dynamically assembles onto the outer membranes of a subpopulation of healthy, elongated mitochondria to promote fission and to inhibit fusion. Following mitochondrial fission, actin disassembles from the fragmented mitochondria, which rapidly re-fuse and reintegrate into the mitochondrial network. Actin subsequently assembles around a distinct, often neighbouring mitochondrial subpopulation and the process repeats. Over $14 \mathrm{~min}$, actin cycles through all mitochondrial subpopulations, locally enhancing mitochondrial dynamics, which facilitates network remodelling and content mixing. Our study thus highlights actin dynamics during mitochondrial fission/fusion and identifies actin cycling as a homeostatic regulator of cellular mitochondrial morphology.

\section{Results}

Actin is associated with mitochondrial subpopulations. To explore the dynamics of actin assembly on mitochondria, we imaged live HeLa cells expressing LifeAct-GFP, a marker that preferentially labels F-actin ${ }^{15}$, and Mito-DsRed2, a mitochondrial matrix marker. As expected, the majority of actin filaments localized to the cell periphery and did not associate with mitochondria (Fig. 1a). However, we were also able to observe F-actin localization to subpopulations of mitochondria (Fig. 1a, Box 1). Using confocal microscopy, we identified clear LifeAct-GFP rings surrounding individual mitochondria (Fig. 1b,c), demonstrating robust recruitment of actin to the outer membrane of these organelles. Three-dimensional renderings of confocal $z$-stacks revealed $\mathrm{F}$-actin cages entirely surrounding subpopulations of cellular mitochondria (Fig. 1d).
At any point in time, we found that LifeAct was recruited to only a subset (on average $23 \%$ ) of the total mitochondrial pool (Fig. 1e). To query the functional consequence of this cytoskeletal association, we compared the size and morphology of actinpositive and actin-negative mitochondria within cells. We found that mitochondria with associated actin were significantly shorter, smaller and less tubular (more circular) than mitochondria without associated actin (Fig. 1f,g,h).

In parallel experiments in untransfected fixed cells, we observed localization of endogenous F-actin (visualized using phalloidin) to TOM20-stained mitochondria in $95.2 \%$ of Hela cells $(n=105$ cells from three independent experiments; Supplementary Fig. 1). Within each cell, we identified F-actin assembly on $22 \%$ of mitochondria (Fig. 1i,j,k and Supplementary Fig. 2a). Consistent with our live-cell data, we found that phalloidin-positive mitochondria were comparatively more fragmented than those not associated with actin (Supplementary Fig. 2b,c,d). In fixed cells, visualization of F-actin recruitment to mitochondria is most apparent in single confocal slices, but is also clearly visible in maximum intensity projections of confocal stacks (Supplementary Fig. 2e).

Actin cycles through mitochondrial subpopulations. Over time, we found that actin filaments did not remain stably associated with the same subset of mitochondria. Instead, we observed actin cycling through different mitochondrial subpopulations. Imaging cells expressing LifeAct-GFP and Mito-DsRed2 over time shows the cycling of polymerized actin from one mitochondrial subpopulation to another (Fig. 2a-c, Supplementary Fig. 3 and Supplementary Movies 1-4). The assembly and disassembly of actin filaments around specific mitochondrial subpopulations is most clearly seen in enlarged images of specific regions of the cell (Fig. 2c) and the corresponding quantification of the overall fluorescence intensity of LifeAct-GFP within these boxed regions (Fig. 2d). Actin remained associated with each subpopulation of mitochondria for $\sim 3-5 \mathrm{~min}$ before depolymerizing (Fig. 2e). On depolymerization, actin subsequently repolymerized around a distinct, usually adjacent, subpopulation of mitochondria (the clockwise movement of the arrows in the time series shown in Fig. 2a and Supplementary Movie 1 is noteworthy), resulting in the robust cycling of actin through the entire mitochondrial network (see model in Fig. 2b). We observed this process of actin cycling in $86.8 \%$ of all cells $(n=114$ cells from 7 independent experiments; Supplementary Movie 5).

To confirm our initial observations of actin dynamics made using LifeAct-GFP, we performed similar imaging experiments using either GFP-actin (Supplementary Fig. 4 and Supplementary Movie 6) or F-tractin-GFP ${ }^{16}$ (Supplementary Movie 7), to visualize actin filaments. With all three probes, we noted very similar dynamics: actin transiently assembled around a subpopulation of mitochondria in the cell and subsequently cycled through distinct mitochondrial subpopulations (Supplementary Figs 3 and 4, and Supplementary Movies 1-6).

Given the persistent unidirectional cycling seen in some cells (Fig. 2a), we examined the directionality of cycling in the cell population. We found some cells exhibited persistent clockwise or anticlockwise cycling, although most commonly we observed a more stochastic result in which the depolymerization of actin filaments from one mitochondrial population was equally likely to be followed by repolymerization around adjacent mitochondria located to either side of the initial subpopulation. We also noted occasional apparent jumps across the cell to non-adjacent populations of mitochondria; however, analysis of time-lapse movies composed of $z$-stack max projections revealed that the observed actin jumping was in fact actin cycling onto intervening adjacent subpopulations of mitochondria either above or below 

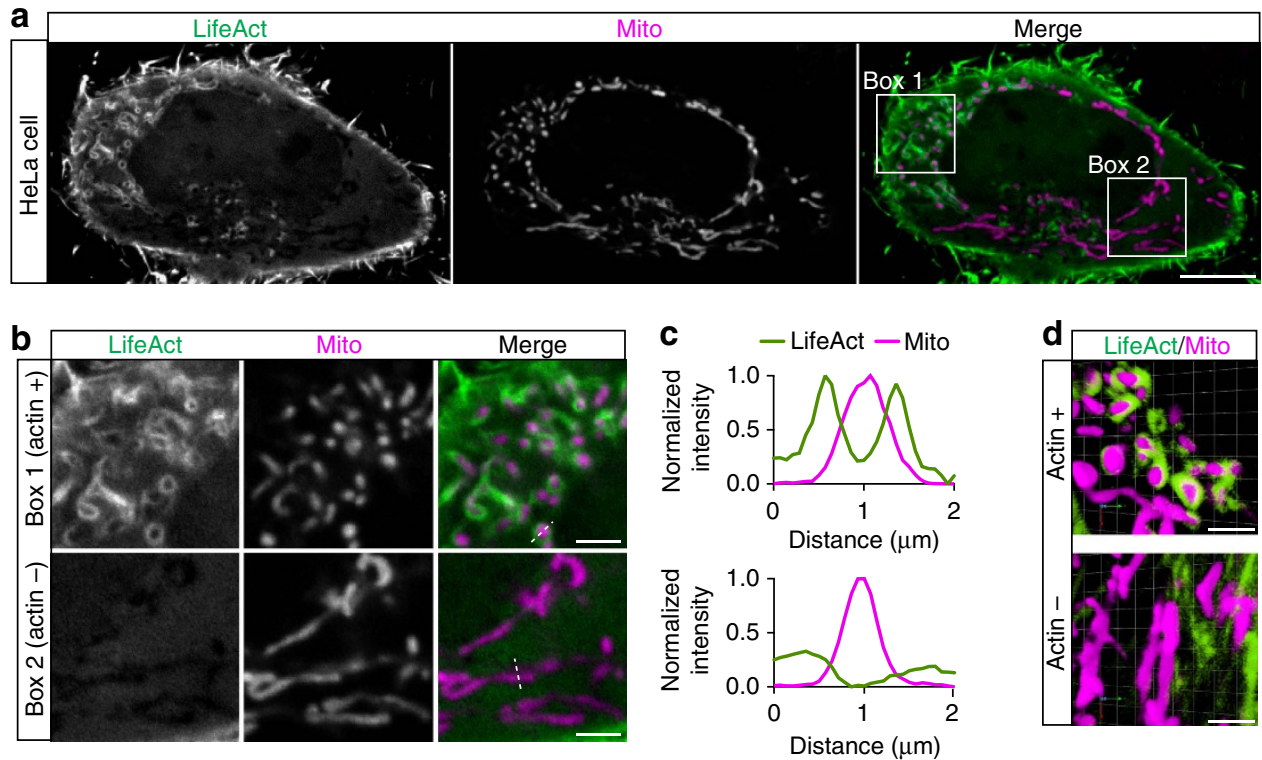

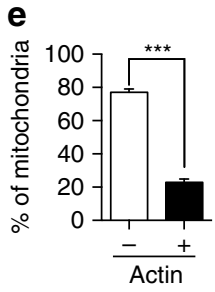

i

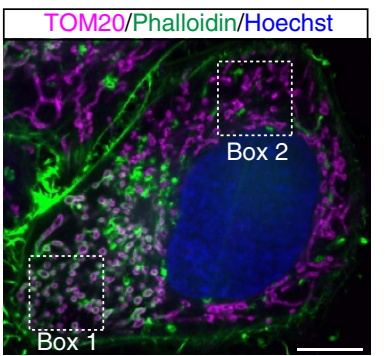

f

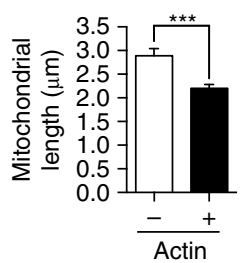

j

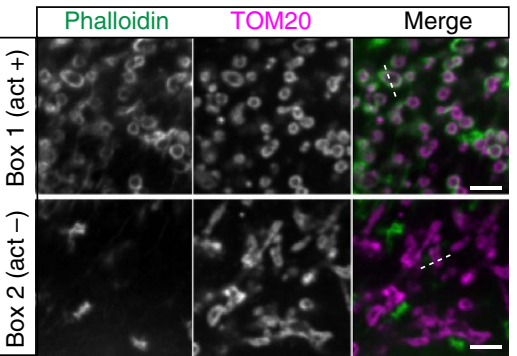

g

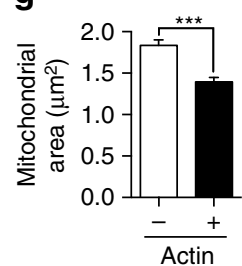

h

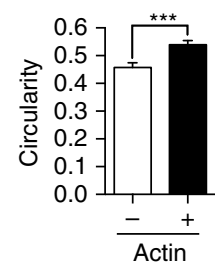

k

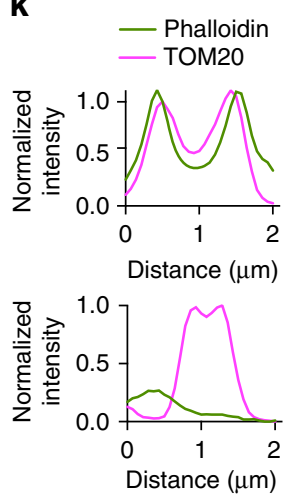

Figure 1 | F-actin localizes to subpopulations of mitochondria. (a) Confocal microscopy image of F-actin (LifeAct-GFP) localization to a subset of Mito-DsRed2-labelled mitochondria in HeLa cells. (b) Enlarged images showing fragmented, actin-positive mitochondria (Box 1) and elongated, tubular mitochondria that have not recruited actin (Box 2). (c) Line scans of actin-positive mitochondria (top) and actin-negative mitochondria (bottom). (d) Three-dimensional renderings of $z$-stack images showing differential morphologies of actin-positive mitochondria (top) and actin-negative mitochondria (bottom) within the same cell. (e) Percentage of actin-positive and actin-negative mitochondria per cell. (f,g) Mitochondria that have recruited actin have decreased length and area. (h) Actin-positive mitochondria have a significantly higher circularity index than actin-negative mitochondria. (i) Confocal image of phalloidin recruitment to mitochondria labelled with anti-TOM2O antibody in a fixed HeLa cell. (j) Enlarged images from the boxed region in $\mathbf{i}$ displaying differential morphologies of phalloidin-positive and phalloidin-negative mitochondria. (k) Line scans of individual mitochondria from the regions shown in $\mathbf{j}$ showing co-localization of the TOM20 outer membrane marker with phalloidin (top) and TOM20 without actin co-localization (bottom). Values represent means \pm s.e.m. ${ }^{\star \star \star} P<0.001$. Scale bars, $10 \mu \mathrm{m}(\mathbf{a}, \mathbf{i}) ; 2.5 \mu \mathrm{m}(\mathbf{b}, \mathbf{d}, \mathbf{j})$. Sample size: (e) 19 cells, (f) 9 cells, (g) 10 cells, (h) 26 cells. $N \geq 3$ independent experiments in all cases.

the nucleus that were outside of the initial confocal plane. The cumulative sampling of mitochondrial subpopulations in a clockwise manner within a single cell is shown in the colourcoded max projection of the distribution of F-actin through time (Supplementary Fig. 3c). The average rate of actin cycling through the cell's total mitochondrial population is $14.0 \pm 0.8 \mathrm{~min}$ per cycle (mean \pm s.e.m.; $n=16$ cells), with a stepwise migration event every $3.7 \pm 0.3 \mathrm{~min}$ (mean \pm s.e.m.; $n=16$ cells).
Next, we asked whether the phenomenon of mitochondrial actin cycling was restricted to HeLa cells or could be seen in other cell types. We imaged LifeAct-GFP and Mito-DsRed2 in COS-7 cells (Supplementary Fig. 5 and Supplementary Movie 8) and noted very similar dynamics of actin assembly and disassembly around mitochondrial subpopulations within a cell, demonstrating that this process is not unique to HeLa cells. We also queried whether similar actin dynamics could be 

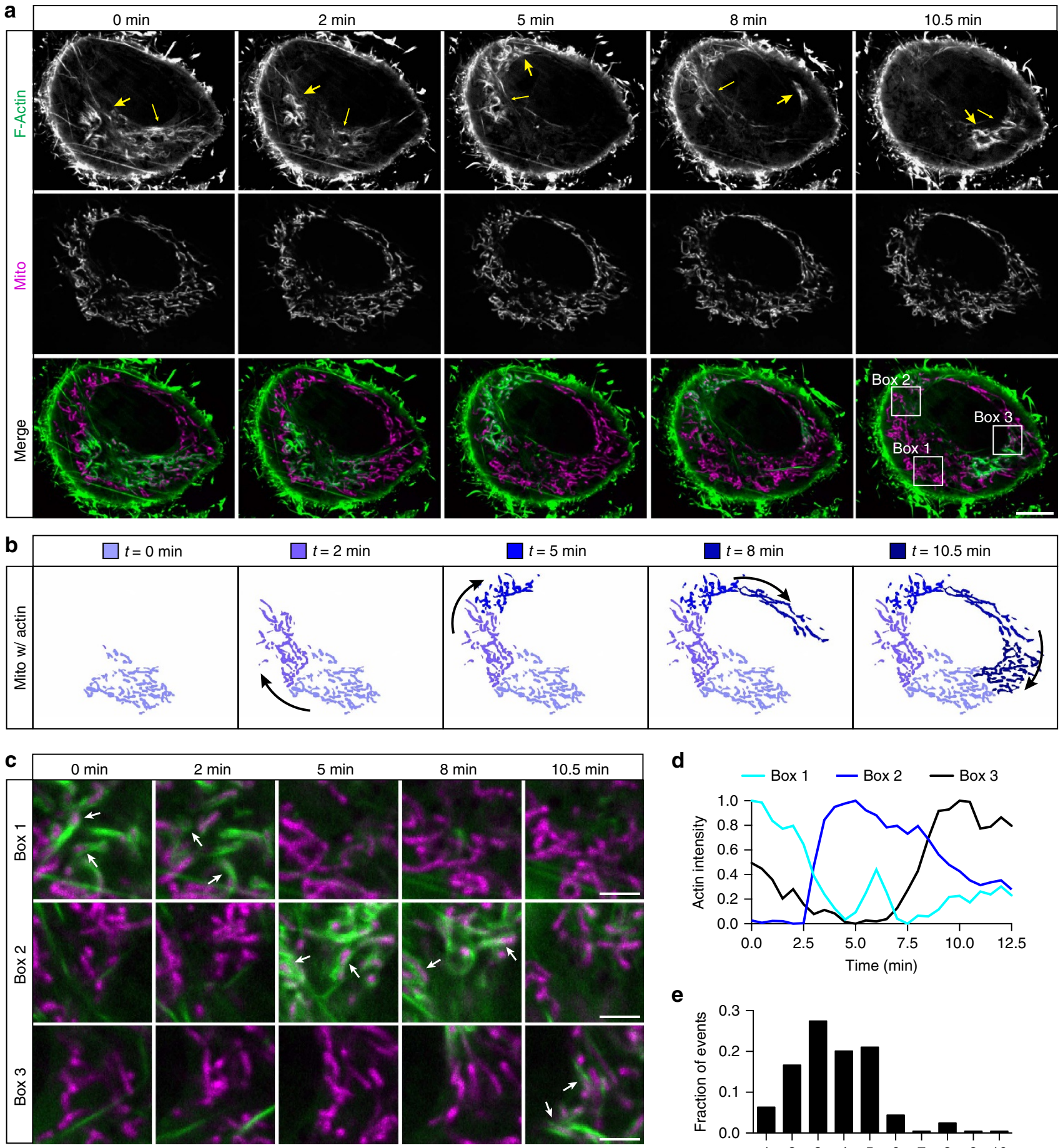

d

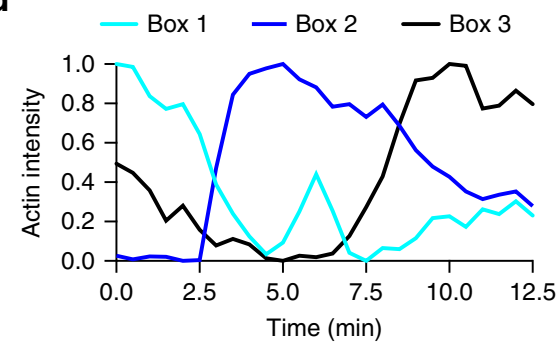

e

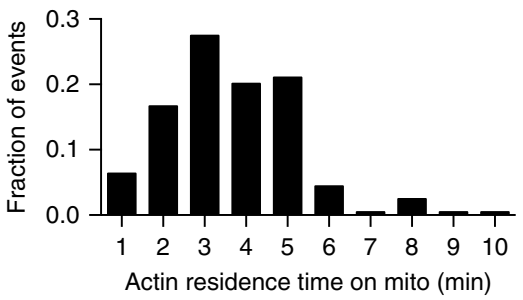

Figure 2 | Actin cycles through mitochondrial subpopulations within the cell. (a) Confocal time series showing F-actin (LifeAct-GFP) cycling through different mitochondrial subpopulations within the cell in a clockwise direction over $10.5 \mathrm{~min}$. Large arrow indicates leading edge of actin-positive mitochondria; small arrow indicates trailing edge of actin-positive mitochondria. (b) Model of actin cycling through mitochondrial subpopulations over time corresponding to (a). (c) Enlarged images of boxes 1-3, showing temporally ordered actin polymerization/depolymerization on individual mitochondria (white arrows). (d) Normalized actin intensities within boxes 1-3 over time. (e) Histogram of F-actin residence time on individual mitochondria during cycling. Values represent means \pm s.e.m. Scale bars, $10 \mu \mathrm{m}$ (a); $2.5 \mu \mathrm{m}$ (c). Sample size: (e) $n=204$ mitochondria from 22 cells; $n \geq 3$ independent experiments.

observed in primary cells by imaging actin and mitochondria over time in normal human epidermal keratinocytes (Supplementary Fig. 6 and Supplementary Movie 9). Again, we saw the periodic assembly and disassembly of actin filaments around mitochondrial subpopulations, indicating that this phenomenon is not related to long-term cell culture or transformation.

Actin cycling is not regulated by membrane potential or ROS. Whole-cell depolarization of mitochondria induced by the 
mitochondrial uncoupler carbonyl-cyanide $m$-chlorophenylhydrazone (CCCP) has been reported to induce fission preceded by the rapid assembly of actin around mitochondria ${ }^{13}$. Consistently, we observed that within 2 min of CCCP addition, actin robustly assembled around the majority of mitochondria (Fig. 3a), effectively inhibiting further cycling of actin through mitochondrial subpopulations. CCCP-induced actin assembly on mitochondria was followed by robust mitochondrial fragmentation within 15 min of CCCP treatment (Fig. 3a).

These observations led us to ask whether the dynamic assembly of actin on specific mitochondrial subpopulations might occur in response to fluctuations in membrane potential. First, we compared actin localization to the intensity of TMRE (tetramethylrhodamine ethyl ester), a fluorescent dye that accumulates in the mitochondrial matrix of healthy, polarized mitochondria but does not remain sequestered in the matrix of depolarized mitochondria. We found that mitochondria were uniformly polarized within each HeLa cell, as has been observed in neurons ${ }^{17}$. We found no difference in the intensity of TMRE staining between the smaller, more circular mitochondria that were positive for actin, and the longer, more tubular mitochondria that showed no actin association (Figs 3b,c); further, we observed no cyclic alterations in TMRE intensity within a given cell over time that would correlate with the cyclic changes in actin ongoing in the cells. Thus, the association of actin filaments with subpopulations of cellular mitochondria is not correlated with fluctuations in their membrane potential.

Next, we asked whether actin cycling onto specific mitochondrial subpopulations occurred in response to production of reactive oxygen species (ROS). We induced localized ROS production within the mitochondrial matrix by expressing the mitochondrially targeted construct mito-KillerRed ${ }^{18}$ and then illuminating cells with $561 \mathrm{~nm}$ light (Supplementary Fig. 7a). Whole-cell illumination effectively bleached the KillerRed fluorescence, indicating activation of ROS production, but had no effect on the dynamic cycling of actin assembly/disassembly through mitochondrial subpopulations in time-lapse imaging for up to $30 \mathrm{~min}$ (Supplementary Fig. 7b).

Instead, the clearest predictor of actin cycling onto a mitochondrial subpopulation was mitochondrial size. When we examined the morphology of mitochondrial subpopulations one minute before actin assembly, we observed that prospective actin-positive mitochondria had an average area of $2.24 \pm 0.13 \mu \mathrm{m}^{2}$ (mean \pm s.e.m.), significantly larger than the average size of all cellular mitochondria $\left(1.77 \pm 0.05 \mu \mathrm{m}^{2}\right.$, mean \pm s.e.m., $P<0.001$; Fig. 3 d).

Actin cycling promotes localized mitochondrial fission. As the dynamics of mitochondrial fission and fusion play an important role in maintaining mitochondrial homeostasis, we investigated a possible role for actin cycling in regulating mitochondrial morphology. Based on our observations that actin preferentially cycles onto elongated mitochondria (Fig. 3d), and that actin-positive mitochondria are more fragmented (Fig. If-h and Supplementary Fig. 2b-d), we hypothesized that actin recruitment promotes rapid mitochondrial fission. To investigate whether actin recruitment precedes and possibly promotes mitochondrial fission events, we closely examined subregions of the cellular mitochondrial network before, during and after actin recruitment.

Before actin recruitment, mitochondria were typically long and tubular (Fig. 4a). Following actin polymerization, mitochondria became constricted (specific sites indicated by yellow circles in Fig. 4a are noteworthy) and then fragmented into smaller pieces. Thus, actin recruitment to each mitochondrial subpopulation resulted in the localized fragmentation of mitochondrial networks a

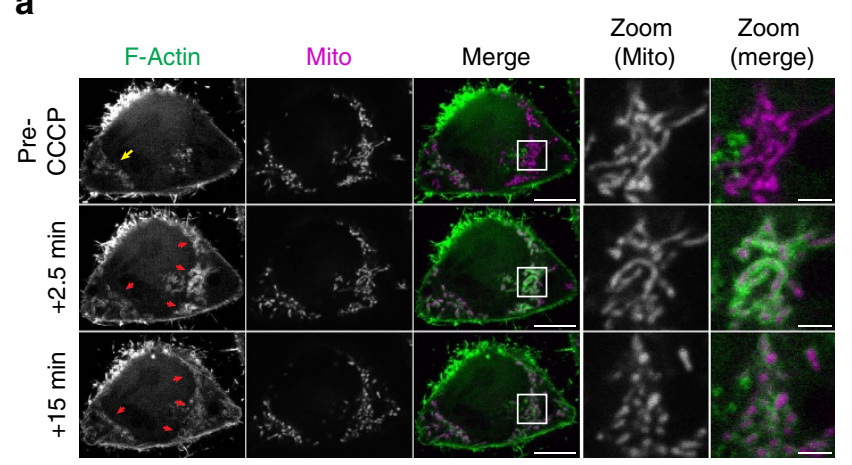

b

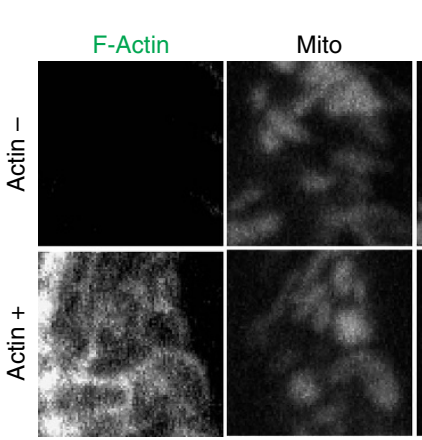

C
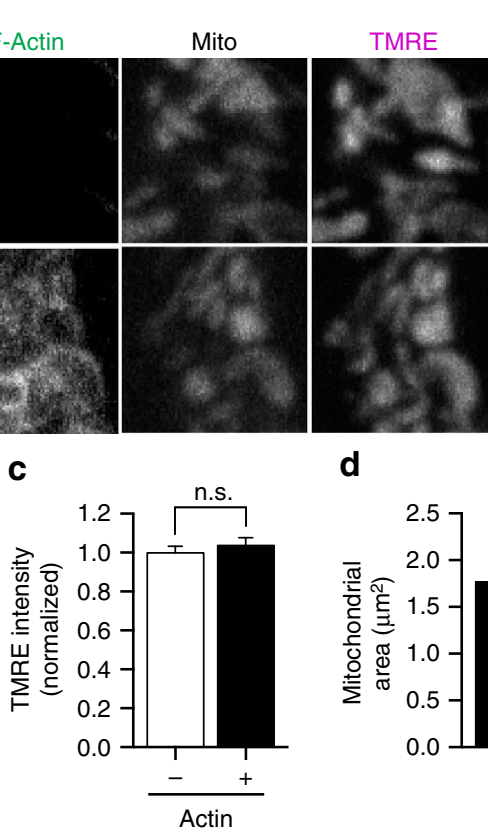

F-Actin/ TMRE

d

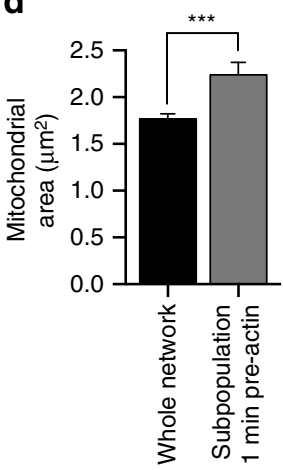

Figure 3 | Actin cycles onto polarized and elongated mitochondria in healthy cells. (a) Time series demonstrating stable recruitment of F-actin (LifeAct-GFP) to mitochondria (Mito-DsRed2) in HeLa cells depolarized by treatment with $20 \mu \mathrm{M}$ CCCP. Before CCCP treatment, cycling actin can be visualized on a subpopulation of HeLa cell mitochondria (yellow arrow, top panel). Within 2.5 min of CCCP treatment, actin was stabilized on $>90 \%$ of all mitochondria (red arrows) and robust fission of all mitochondria was observed by 15 min post CCCP treatment. (b,c) Untreated cells expressing LifeAct-GFP and Mito-BFP display no significant difference in TMRE intensity between actin-positive and actin-negative mitochondria. (d) One minute before actin recruitment, mitochondria targeted for actin assembly are significantly larger than average mitochondria within the cell. Values represent means \pm s.e.m. ${ }^{\star \star \star} P<0.001 ;$ NS, not significant. Scale bars, $10 \mu \mathrm{m}$ (a full size); $2.5 \mu \mathrm{m}$ (a zoom, b). Sample size: (c) 50 mitochondria from 10 cells per condition; (d, whole network) 27 cells; (d, actin-targeted subpopulation) 26 cells; $n \geq 3$ independent experiments in all cases.

(Supplementary Movie 10). In contrast, mitochondria that had not recruited actin within the same timeframe maintained their characteristic tubular and interconnected morphology.

We directly compared the kinetics of actin recruitment to changes in mitochondrial size (area and length) within specific regions of the cell (Fig. $4 b, c)$. As actin intensity increased, the average individual mitochondrial size significantly decreased over a time scale of $4 \mathrm{~min}$. Next, we examined the effects of localized actin assembly on the complexity of the mitochondria network. 

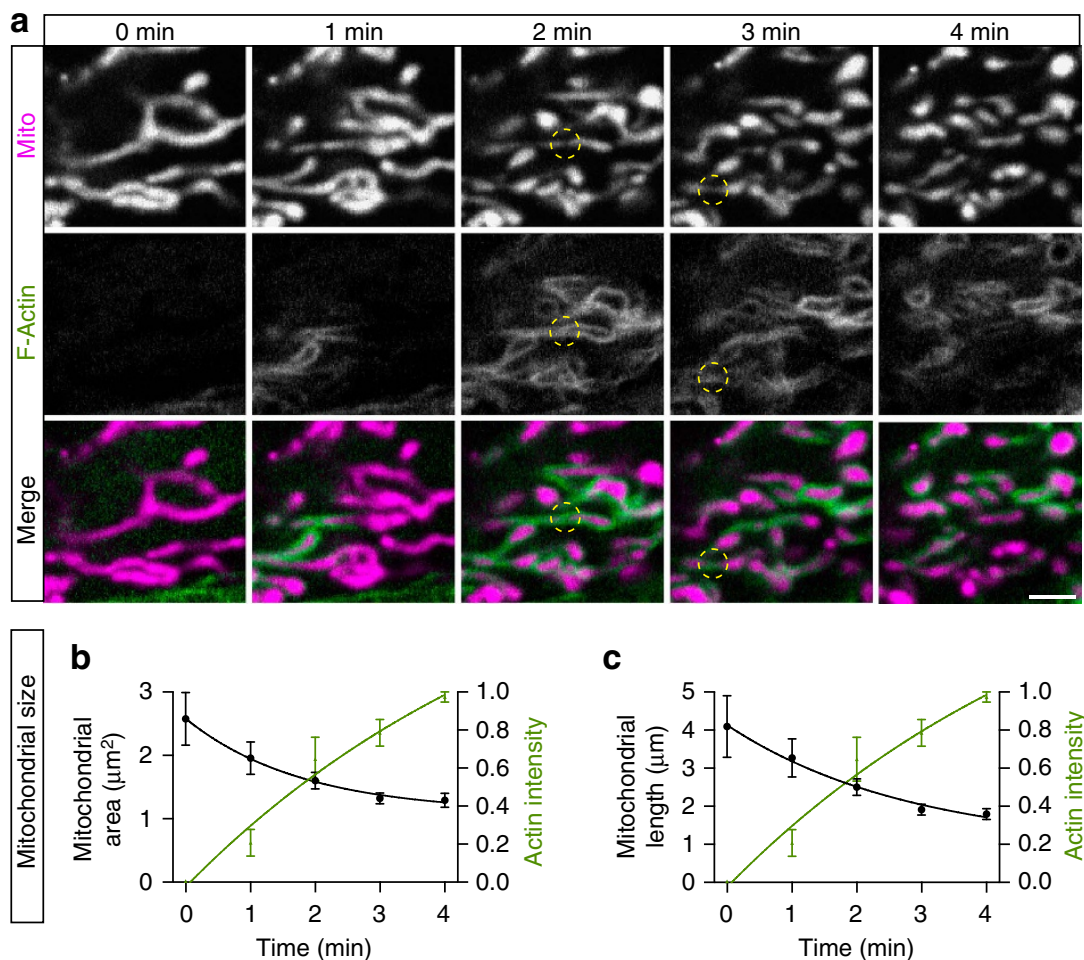

C
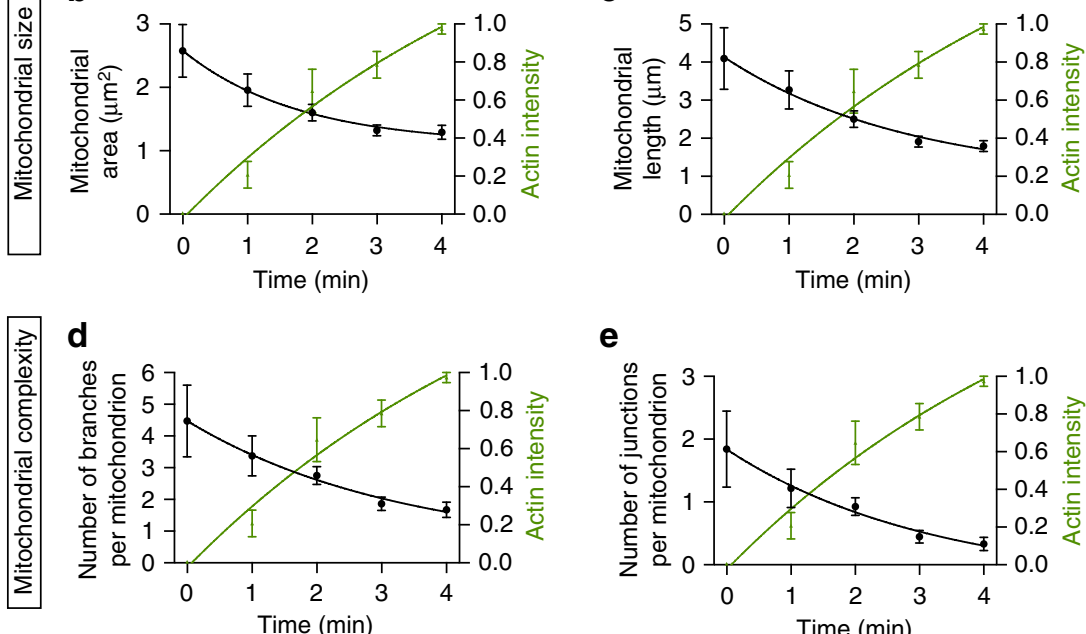

e
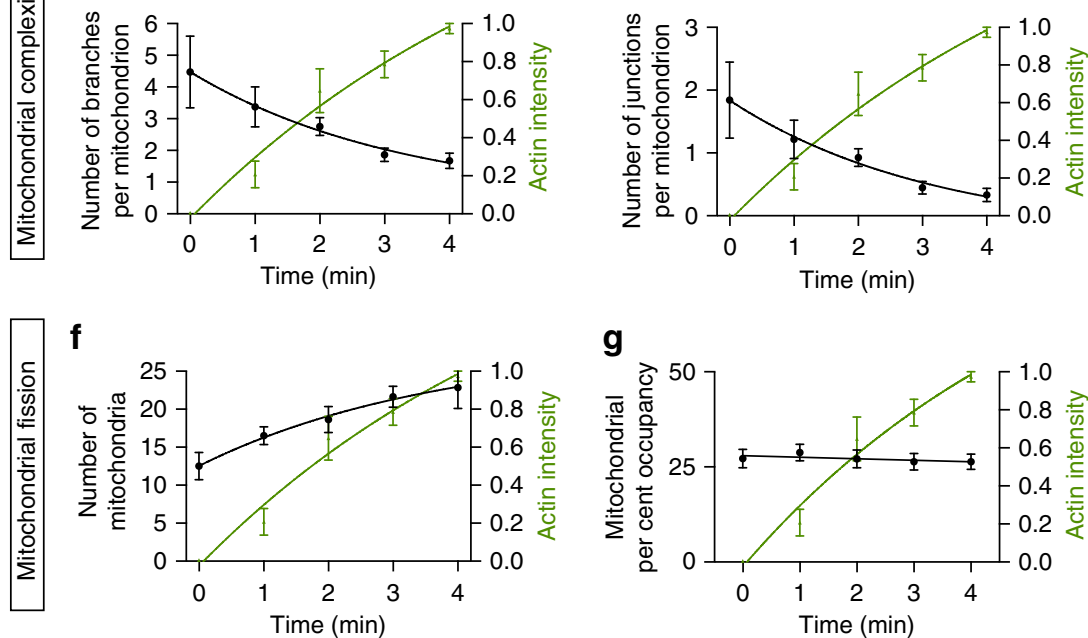

Figure 4 | The mitochondrial network fragments in regions of focal actin assembly. (a) Confocal time series of F-actin (LifeAct-GFP) cycling onto elongated, tubular mitochondria (Mito-DsRed2) in a HeLa cell. Over 4 min, actin-positive mitochondria become increasingly fragmented. Yellow circles indicate sites of mitochondrial constriction. $(\mathbf{b}, \mathbf{c})$ Mitochondrial area and length decrease as actin intensity increases within a defined ROI (see Methods for details). (d,e) Actin recruitment to mitochondria is linked to changes in mitochondrial shape complexity, including decreased branches and junction points per mitochondrion. $(\mathbf{f}, \mathbf{g})$ Actin cycling onto mitochondria is associated with increased mitochondrial number, but no change in the overall mitochondrial per cent occupancy within the fixed ROI. Values represent mean \pm s.e.m. Scale bar, $2.5 \mu \mathrm{m}$ (a). Sample size: (b-g) 6 cells; $n=3$ independent experiments.

Over the same time scale and within the same localized region of the cell, we found that the increase in actin intensity was correlated with a decrease in the connectedness of the mitochondrial network, assessed as the number of branches per mitochondrion (Fig. 4d) and the number of junctions per mitochondrion (Fig. 4e; see Methods for details on the segmentation analysis used to assess these parameters).

We next assessed the frequency of mitochondrial fission in relation to actin association. The increased actin intensity, indicative of dynamic actin assembly around a subpopulation of cellular mitochondria, was closely correlated with a doubling in the number of mitochondria undergoing fission within this mitochondrial subpopulation (from $12.5 \pm 1.8$ to $22.8 \pm 2.7$; Fig. 4f). To demonstrate that these changes were due to remodelling of the resident mitochondrial population rather than movement of mitochondria into or out of the region of interest (ROI), we calculated the mitochondrial per cent occupancy for each ROI and found no significant change over time (Fig. 4g). Similarly, we tracked actin-associated mitochondrial fragmentation in $z$-stack three-dimensional projections (Supplementary Fig. 8), confirming that the observed fission is not due to the rotation or translocation of mitochondria in the $z$-axis.

Finally, to confirm that our actin markers were not contributing to the dynamic mitochondrial re-organization we observed 
upon actin recruitment, we performed live-cell imaging of cells expressing only Mito-DsRed2. Even in the absence of actin markers, we observed distinct subregions of the mitochondrial network undergoing fragmentation concurrent with continued elongation of the mitochondrial network throughout the rest of the cell (Supplementary Fig. 9). Thus, our live-imaging studies demonstrate that actin polymerization around mitochondrial subpopulations leads to a local enhancement of the frequency of fission, resulting in localized mitochondrial fragmentation and decreased interconnectivity.

Actin polymerization inhibits mitochondrial fusion. Assembly of actin around mitochondrial subpopulations clearly enhances fission, but we wondered whether this assembly might also inhibit fusion. Specifically, the formation of F-actin networks around mitochondria might decrease organelle motility and serve as a barrier to fusion with neighbouring mitochondria. We found that, indeed, mitochondrial motility was significantly decreased when actin was assembled on mitochondria as compared with the motility of mitochondria without co-localized actin: we found the mean displacements over time for actin-positive and actin-negative mitochondria were $0.27 \pm 0.04 \mu \mathrm{m} \mathrm{min}^{-1}$ and $0.62 \pm 0.07 \mu \mathrm{m} \mathrm{min}^{-1}$, respectively (mean \pm s.e.m., $P<0.001$, $n=16$ cells per condition from 3 independent experiments).

Next, we asked whether actin disassembly was accompanied by an increase in the frequency of mitochondrial fusion. We focused on mitochondrial subpopulations enriched for actin and assayed for effects on mitochondrial morphology as the associated actin filaments disassembled (Fig. 5a). Quantitative analysis demonstrates that the decline in the fluorescence intensity of LifeAct-GFP over a $4 \mathrm{~min}$ time course of actin disassembly is correlated with increased mitochondrial area and length (Fig. 5b,c and Supplementary Movie 11). The interconnectedness of the mitochondrial network also increased as actin disassembled, as we noted significant increases in both the number of branches per mitochondrion and the number of junctions per mitochondrion (Fig. 5d,e). Finally, there was a decrease in the total number of individual mitochondria (Fig. 5f), but no change in the per cent occupancy within the examined ROI (Fig. 5g), consistent with an enhancement of fusion once actin disassembles from the given mitochondrial subpopulation. Tracking individual organelles, we observed that daughter mitochondria from fission events frequently moved in opposite directions before fusing with distinct, neighbouring mitochondria, thus promoting content mixing and network remodelling (Supplementary Fig. 10).

Actin cycling requires Arp2/3 and formins. The dynamics of actin cycling observed in live cells strongly suggest that polymerization of new, organelle-associated actin filaments is required. Therefore, this process should be inhibited by treatment with the actin-depolymerizing drug, Latrunculin B. Before treatment, we noted robust actin recruitment to a subset of mitochondria (Fig. 6a, left panel). After 20 min of Latrunculin B treatment, F-actin was not observed in association with any mitochondrial population in the cell (Fig. 6a,b).

Next, we asked whether the assembly of actin filaments onto mitochondria was nucleated by either Arp $2 / 3$ or formins. Following a $1 \mathrm{~h}$ treatment of HeLa cells with the Arp2/3 inhibitor CK-666, actin cycling on mitochondrial subpopulations was completely abolished and actin filaments were no longer associated with mitochondria (Fig. 6b). As recent work has implicated the formin INF2 and the formin-binding protein Spire $1 \mathrm{C}$ in mitochondrial fission ${ }^{10,12}$, we also tested whether formin activity is required for dynamic actin cycling. We treated cells with the small molecule formin inhibitor SMIFH2 for $1 \mathrm{~h}$. Again, we noted the complete inhibition of dynamic actin cycling and the loss of actin association with mitochondria (Fig. 6b). Thus, both Arp2/3 and formins contribute to the dynamic formation of the mitochondria-associated actin filaments observed in our live-cell assays and inhibition of either nucleation mechanism is sufficient to block mitochondrial-associated actin cycling.

Fission of actin-positive mitochondria requires Drp1. Actin recruitment to mitochondria leads to enhanced fission, as shown above. This led us to ask whether the observed fission of actin-positive mitochondria is dependent on the canonical fission machinery. We first investigated the localization of Drp1, the dynamin-related GTPase that regulates mitochondrial fission, during actin cycling. In HeLa cells transfected with GFP-Drp1, we did not observe preferential recruitment of Drp1 to mitochondria positive for actin (Drp1 puncta on actin-positive mitochondria $1.21 \pm 0.16$ puncta per $\mu \mathrm{m}$, Drp1 puncta on actin-negative mitochondria $1.23 \pm 0.20$ puncta per $\mu \mathrm{m}$ (mean \pm s.e.m., $P=0.93$, $n=11$ cells from three independent experiments)). In fixed cells we observed comparable patterns of Drp1 staining on both phalloidin-positive and phalloidin-negative mitochondria, indicating that endogenous Drp1 is not preferentially recruited to actin-positive mitochondria.

Next, we asked whether Drp1 activity is necessary for actininduced fission. To investigate this question, we transfected HeLa cells with Mito-DsRed2, LifeAct-GFP and either an empty vector control or Drp1-K38A, a dominant-negative mutant that has been demonstrated to inhibit Drp1 activity ${ }^{19}$. Consistent with previous reports, expression of Drp1-K38A resulted in profound mitochondrial elongation and hyperfusion. In both control and Drp1-K38A-expressing cells, we identified actin assembly onto and disassembly off of subpopulations of mitochondria (Fig. 6c,d). Further, we found that expression of the dominantnegative Drp1 construct did not affect the mean time required for actin to cycle onto all mitochondrial subpopulations (Fig. 6e). However, mitochondria in cells expressing Drp1-K38A failed to undergo robust mitochondrial fission on actin assembly. Quantitative analysis of $z$-stack projections indicates a significant increase in mitochondrial fission on actin recruitment to mitochondria in control cells, but only a modest change in mitochondrial number on actin recruitment to mitochondria in Drp1-K38A-expressing cells (Fig. 6f). Similarly, we identified a $63 \%$ decrease in mitochondrial length on actin recruitment in control cells and only a $25 \%$ decrease in cells transfected with Drp1-K38A (Fig. 6g). Consistent with this observation, cells treated with the Drp1 inhibitor ${ }^{20}$ Mdivi-1 did not display robust mitochondrial fission on actin assembly (Supplementary Fig. 11). Therefore, actin cycles through mitochondrial subpopulations independently of Drp1 activity, but Drp1 is required for efficient fragmentation and remodelling of the mitochondrial network following localized actin assembly.

Actin assembles at ER-mitochondria contacts. Recent work has identified key roles for ER-mitochondrial contact sites in lipid exchange, calcium signalling as well as the initiation of mitochondrial fission ${ }^{9,21}$. Thus, we investigated whether actin preferentially cycles onto these inter-organelle junctions. Before actin recruitment, we frequently observed the ER in the vicinity of mitochondria (Fig. 7a). As actin polymerized on elongated mitochondria, we identified uniform actin recruitment to the outer mitochondrial membrane with specific enrichment of actin at sites of ER-mitochondria overlap (Fig. 7a,b). Line-scan analysis indicated close apposition of actin with $\mathrm{ER}$ at sites of mitochondrial constriction before fission (Fig. 7b,c). In threedimensional renderings of these ER-mitochondria contact sites, 


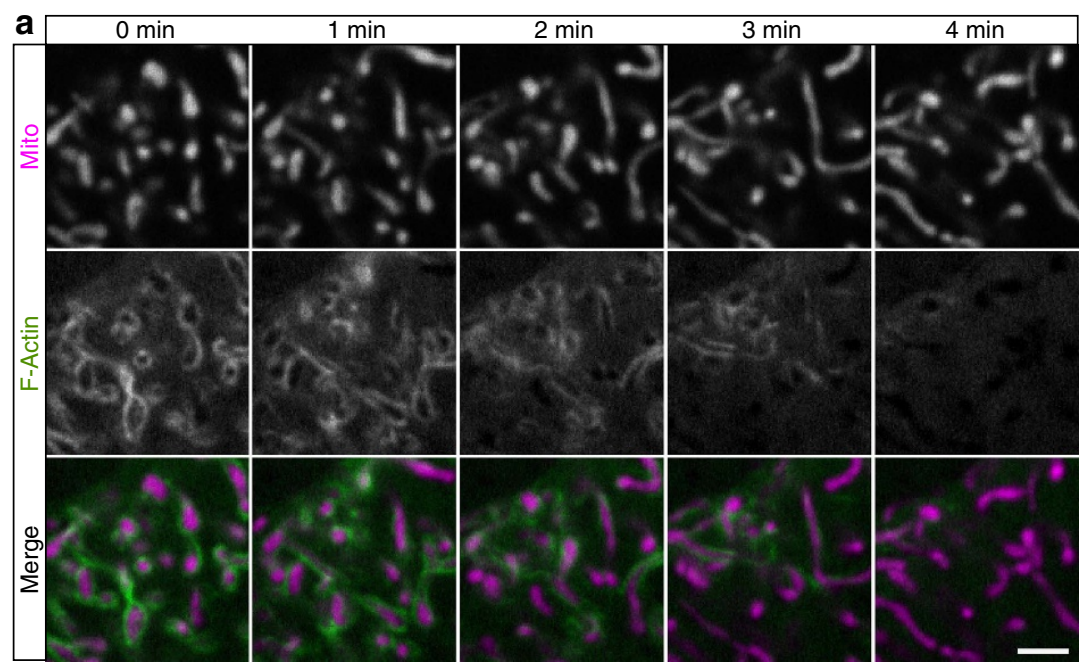

b

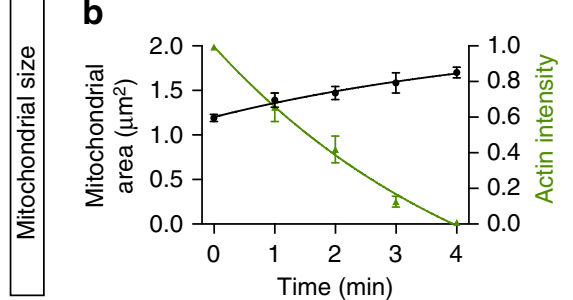

d

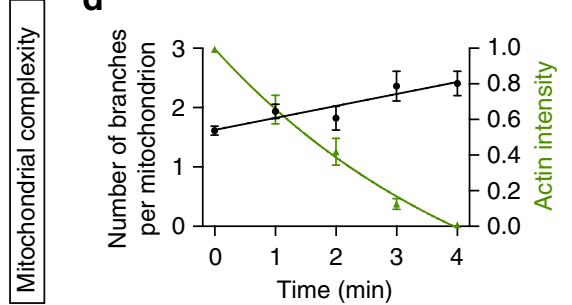

f

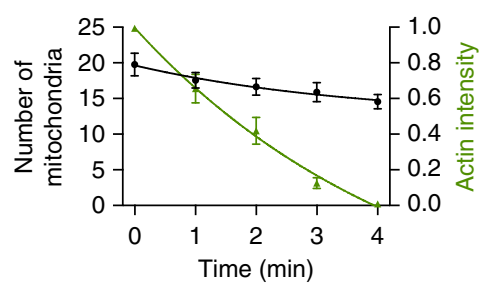

C

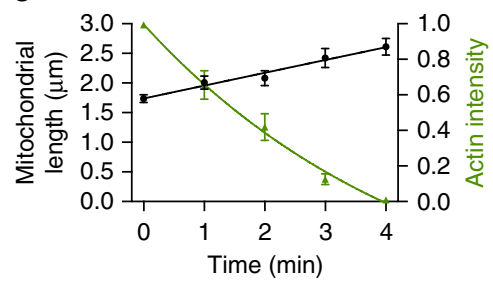

e

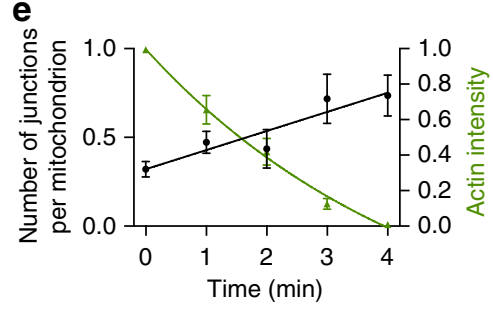

g

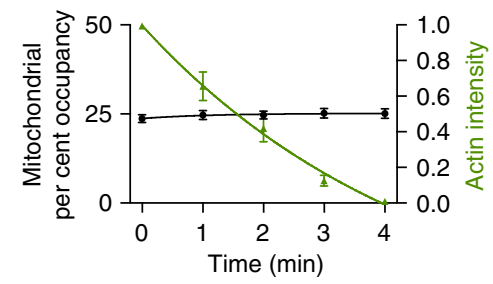

Figure 5 | Mitochondria undergo rapid growth on actin disassembly. (a) Confocal time series showing actin (LifeAct-GFP) depolymerization from a subpopulation of fragmented, rounded mitochondria (Mito-DsRed2). On actin disassembly, mitochondria undergo rapid growth and fusion.

$(\mathbf{b}, \mathbf{c})$ Mitochondrial area and length increase as actin intensity decreases. (d,e) Actin depolymerization augments mitochondrial complexity, resulting in an increased number of mitochondrial branches and junctions. (f,g) Actin disassembly leads to a decreased number of mitochondria, but has little effect on total mitochondrial percent occupancy, suggesting that fusion is enhanced on actin depolymerization. Values represent mean \pm s.e.m. Scale bar, $2.5 \mu \mathrm{m}$ (a). Sample size: (b-g) 9 cells; $n \geq 3$ independent experiments.

we see actin specifically enriched at points of mitochondrial constriction, where we observe the initiation of a fission event (Fig. 7d). Thus, dynamic actin cycling may facilitate mitochondrial fission through the induction of ER-dependent mitochondrial constriction. This interpretation is consistent with recent work demonstrating a role for the mitochondrially localized Spire 1C and ER-localized INF2 in the formation of mitochondrial constrictions ${ }^{10,12}$.

Effects of actin cycling on the mitochondrial network. Our data fit a model in which the active polymerization of actin on a mitochondrial subpopulation promotes fission, whereas actin disassembly allows fusion, locally restoring the integrity of the mitochondrial network (Fig. 8a). To quantitatively test this model, we directly compared mitochondrial length, number of branches and overall mitochondrial number in subcellular regions of comparable size: (1) before and after actin polymerization, (2) before and after actin depolymerization and (3) in cellular regions in which no actin cycling was observed over a 4 min time period. As shown in Fig. 8b,c, active polymerization of actin led to a significant decrease in mitochondrial length within $4 \mathrm{~min}$, with a corresponding increase in mitochondrial number. In contrast, actin depolymerization was followed by an increase in mitochondrial length and a decrease in mitochondrial number 

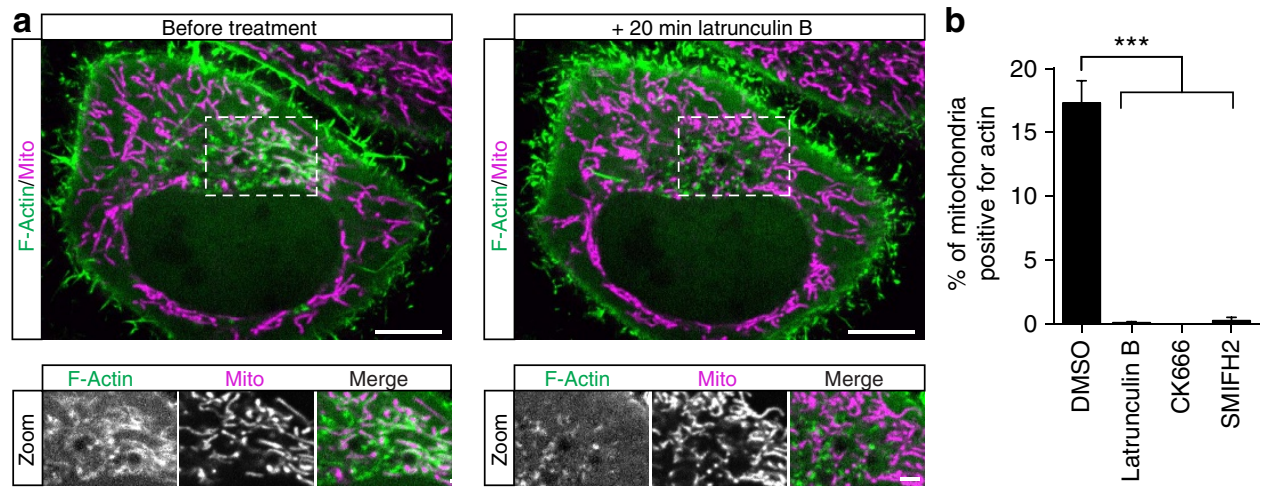

C

Control

d
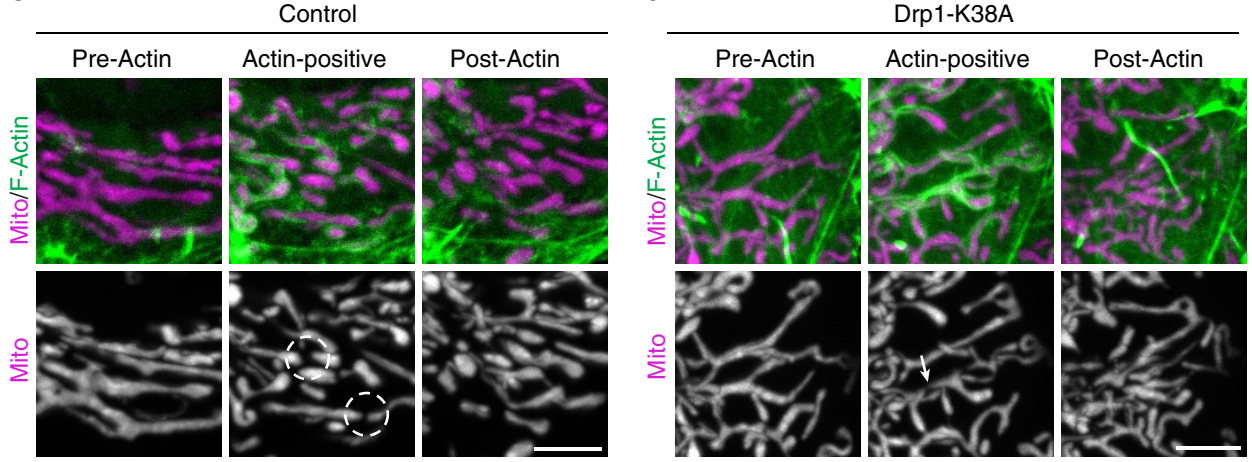

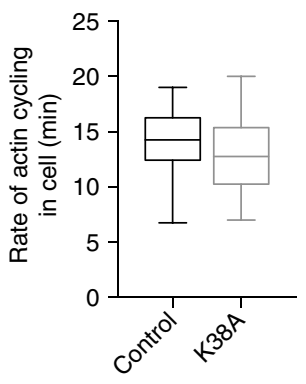

f

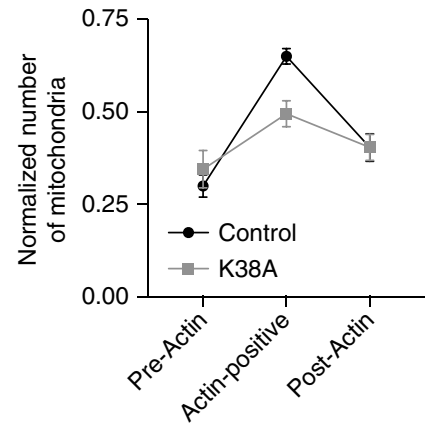

g

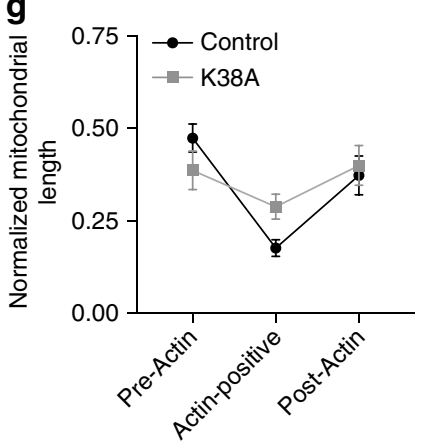

Figure 6 | Actin cycling is dependent on actin polymerization by Arp2/3 and formins but not Drp1 activity. (a) Confocal images of F-actin (LifeAct-GFP) localized to a mitochondrial subpopulation (Mito-DsRed2) during constitutive cycling (left) in a HeLa cell. Actin recruitment to mitochondria was abolished on 20 min treatment with the actin depolymerizing drug Latrunculin B $(2 \mu \mathrm{M})$ (right). (b) F-actin assembly on mitochondria was blocked in cells treated with $2 \mu \mathrm{M}$ Latrunculin B (30 min), $84 \mu \mathrm{M}$ CK-666 (1 h), or $25 \mu \mathrm{M} \mathrm{SMIFH2} \mathrm{(1} \mathrm{h).} \mathrm{(c,d)} \mathrm{Maximum} \mathrm{intensity} \mathrm{projections,} \mathrm{indicating} \mathrm{F-actin} \mathrm{(LifeAct-GFP)}$ assembly onto and disassembly off of mitochondria (Mito-DsRed2) in HeLa cells transfected with either (c) an empty vector or (d) Drp1-K38A. For three-dimensional renderings of mitochondria undergoing fragmentation in control cells, see Supplementary Fig. 8. (e) Mean duration of actin cycling through the entire mitochondrial network in control and Drp1-K38A-expressing cells. $(\mathbf{f}, \mathbf{g})$ Normalized number and length of mitochondria before, during and after actin ssembly in either control or Drp1-K38A-expressing cells. Values represent mean \pm s.e.m. ${ }^{\star \star \star} P<0.001$. Scale bars, $10 \mu \mathrm{m}(\mathbf{a}$ full size); $5 \mu \mathrm{m}$ (c,d); $2.5 \mu \mathrm{m}$ (a, zoom). Sample size: (b, dimethyl sulfoxide, DMSO) 9 cells, (b, Latrunculin) 91 cells, (b, CK-666) 101 cells, (b, SMIFH2) 24 cells, (e) 16 cells, (f,g control) 15 cells, (f,g, K38A) 14 cells; $n \geq 3$ independent experiments in all cases.

over a similar $4 \mathrm{~min}$ time course. Of note, in the absence of associated actin filaments, we noted a steady growth in mitochondrial length over the same 4 min window and a small decrease in the number of mitochondria, suggesting that in the absence of external factors such as actin dynamics, fusion may dominate over fission.

To assess the effects of actin assembly/disassembly on the interconnectedness of the mitochondrial network, we measured the average number of branches per mitochondrion over time (Fig. 8d). Again, actin polymerization led to a significant fragmentation of the network, which was reversed upon actin depolymerization. As with mitochondrial length, we saw that in the absence of associated actin filaments, there is a tendency for the mitochondrial network to increase in complexity over time.

\section{Discussion}

In this study, we used live-cell imaging to examine actin dynamics during mitochondrial fission and fusion. We propose that cyclic actin assembly/disassembly on mitochondrial subpopulations acts to regulate steady-state mitochondrial morphology. Actin cycles through mitochondrial subpopulations in the cell, assembling on the outer membrane of elongated mitochondria to promote fission events and transiently inhibit mitochondrial fusion and motility. Subsequent disassembly of actin facilitates reconfiguration of the mitochondrial network and mixing of mitochondrial contents. Cycles of actin assembly and disassembly survey and regulate the morphology of the total mitochondrial population within the cell in $14 \mathrm{~min}$. In contrast to the chiral symmetry that the actin cytoskeleton 

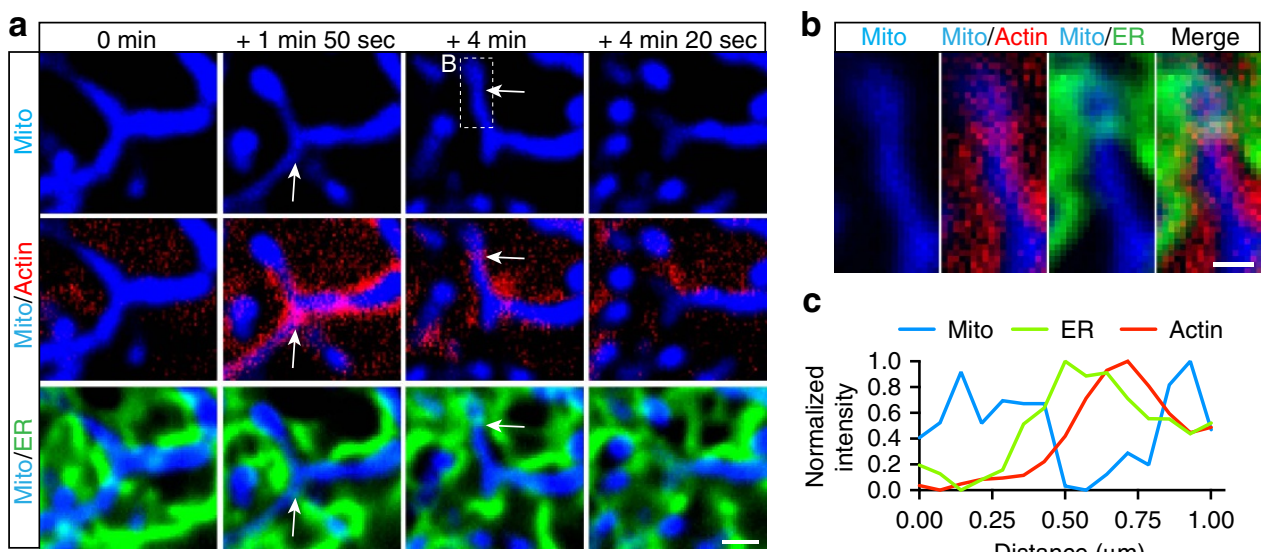

C
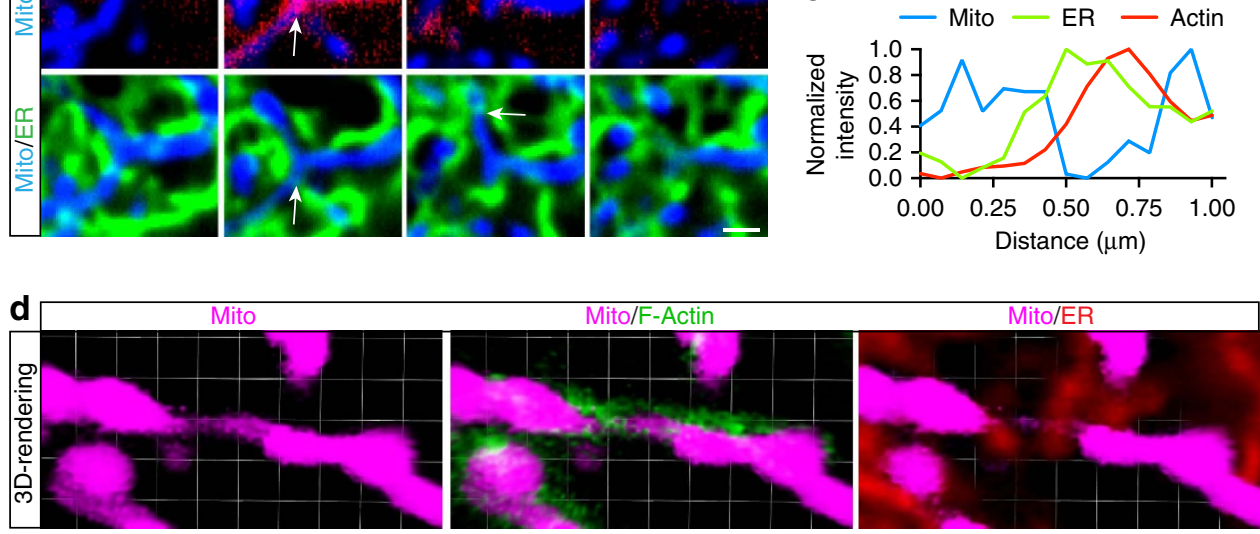

Figure 7 | Actin assembles at ER-mitochondria contact sites. (a) Confocal image of GFP-actin (red) recruitment to elongated, Mito-SNAP-labelled mitochondria (blue) in a HeLa cell. Actin assembly is enriched at sites of ER tubule (DsRed2-ER, green) overlap with mitochondria. Arrows indicate regions of Actin/ER co-localization at prospective sites of mitochondrial fission. (b) Enlarged image of Box B ( +4 min), demonstrating co-localization of actin and ER at the site of mitochondrial fission. (c) Line scan indicating overlapping peak actin and ER intensity at site of mitochondrial constriction in (b). (d) Three-dimensional rendering of F-actin (LifeAct-GFP) and ER-tubules (DsRed2-ER) assembled on a constricted HeLa cell mitochondrion (Mito-SNAP). Scale bars, $1 \mu \mathrm{m}$ (a), $0.5 \mu \mathrm{m}$ (b) and $0.75 \mu \mathrm{m}$ per unit (d).

\section{a}

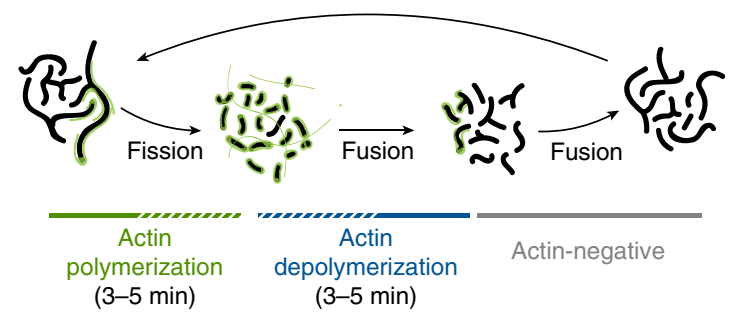

b

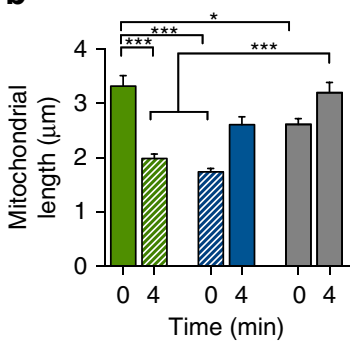

c

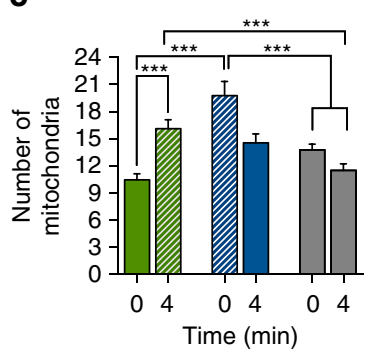

Actin polymerization

Actin depolymerization

d

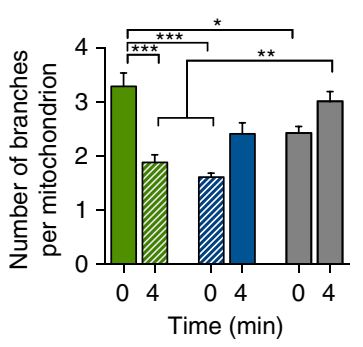

Figure 8 | Actin cycling through mitochondrial subpopulations regulates steady-state mitochondrial morphology. (a) Model: actin polymerizes on a subpopulation of elongated mitochondria, inducing robust fragmentation. After 3-5 min, actin then depolymerizes, allowing the fragmented mitochondria to refuse and slowly increase in size and complexity. Actin-negative mitochondria slowly continue to grow until they become locally hyperfused, at which point actin is recruited and the cycle repeats. (b) Mitochondrial size decreases with actin recruitment and recovers on subsequent actin depolymerization. (c) Actin polymerization induces rapid mitochondrial fission, whereas depolymerization promotes slow fusion. (d) Mitochondrial complexity decreases with actin recruitment and recovers on actin depolymerization. Values represent mean \pm s.e.m. ${ }^{\star \star \star} P<0.001$, ${ }^{\star \star} P<0.01$ and ${ }^{\star} P<0.05$. Sample size: (b-d, actin polymerization) 26 cells, (b-d, actin depolymerization) 9 cells, (b-d, actin negative) 32 cells; $n \geq 3$ independent experiments in all cases.

can adopt in developing fibroblasts ${ }^{22}$, we found that actin showed no directional bias in its recruitment to mitochondrial subpopulations. Further, we found no evidence for fluctuations in mitochondria membrane potential or ROS production that might locally promote actin assembly around mitochondrial subpopulations.

At any point in time, actin filaments are associated with $\sim 20 \%$ of cellular mitochondria. This association is transient, as actin 
remains assembled on mitochondrial subpopulations for 3-5 $\mathrm{min}$. During this period, individual organelles exhibit enhanced fission and suppressed fusion, leading to localized fragmentation of the mitochondrial network. Consistent with previous reports ${ }^{9,12}$, we frequently observe fission events in the vicinity of mitochondria-ER contacts.

Fragmentation of actin-positive mitochondria is dependent on Drp1 activity, as inhibition of Drp1 by either treatment with the inhibitor Mdivi-1 or expression of a dominant-negative Drp1-K38A blocks the local remodelling of actin-positive mitochondria. Of note, inhibition of Drp1 has no effect on the rate of actin cycling through the mitochondrial network, indicating that actin assembly and disassembly from mitochondrial subpopulations is not dependent on successful mitochondrial fission. Using both fixed and live-cell techniques, we observed no differences in the localization or density of Drp1 puncta between actin-negative and actin-positive mitochondria. However, Drp1 puncta on actin-positive mitochondria may be differentially primed for fission through posttranslational modifications ${ }^{23,24}$, association with outer-membrane fission receptors such as Mff or Fis1 or even simply through binding to actin itself ${ }^{8,14}$.

Actin assembly on mitochondria results in a $\sim 50 \%$ reduction in mitochondrial motility. This reduction in motility may contribute to the inhibition of mitochondrial fusion during local network remodelling. Specifically, decreased mitochondrial velocity reduces the probability of inter-mitochondrial contact events, which are necessary for successful fusion ${ }^{25}$. In addition, the formation of an actin cage around mitochondria may obstruct the association of mitofusin proteins between adjacent mitochondria, further potentiating the fusion block. On actin disassembly from a mitochondrial subpopulation, the organelles rapidly undergo fusion to mix their contents and restore a tubular mitochondrial network.

We found that F-actin recruitment to mitochondria is dependent on both Arp2/3 and formins. Curiously, inhibition of either actin-filament nucleator entirely abolished actin cycling through mitochondrial subpopulations, suggesting that Arp $2 / 3$ and formin family proteins work together to facilitate actin polymerization on the mitochondrial outer membrane. Work in budding yeast has identified Arp2/3-dependent actin clouds around fragmenting mitochondria ${ }^{26}$. In addition, Arp2/3 has been identified on the mitochondrial outer membrane of mammalian cells, where it regulates mitochondrial morphology ${ }^{13}$. Recently, the formin-binding protein Spire1C was identified on the mitochondria outer membrane, where it works in close association with the ER-localized formin INF2 to regulate mitochondrial length ${ }^{12}$. Actin depolymerization by Latrunculin $\mathrm{B}$, as well as depletion of Arp2/3 or certain formins results in robust mitochondrial elongation ${ }^{10,12,13}$. Moving forward, it will be interesting to examine the signalling pathways responsible for activation of actin-nucleating proteins on elongated mitochondria, leading to localized filament assembly, as well as to investigate actin filament organization at higher resolution.

We propose that actin cycling through mitochondrial subpopulations serves as a surveillance method to identify and fragment regions of elongated mitochondria. This hypothesis is consistent with previous observations indicating that elongated mitochondria are more likely to undergo fission ${ }^{25}$. Regional regulation of mitochondrial dynamics may confer a number of advantages in the maintenance of mitochondrial networks. First, localized mitochondrial network remodelling could be important for mitochondrial biogenesis and the maintenance and distribution of mitochondrial DNA nucleoids 27,28 . Second, mitochondrial network remodelling may facilitate efficient transport of organelles through the cytoplasm, as previous work from our lab has demonstrated that interactions between organelles can restrict organelle trafficking ${ }^{29}$. An intricately connected mitochondrial network might have the same effect, such that local remodelling would be permissive for intracellular trafficking. Third, local remodelling of mitochondrial subpopulations allows for the fragmentation of individual, overgrown mitochondria without substantially altering the integrity of the mitochondrial network as a whole. As networkwide mitochondrial elongation has been linked to cellular senescence $^{30}$, actin cycling may be a highly important homeostatic function to offset premature senescence. Fourth, actin polymerization onto only one mitochondrial subpopulation at a time ensures that the cellular actin pool is not depleted, consistent with observations that most dynamic cellular actin is enriched at the cortex rather than in close association with mitochondria. Finally, constitutive actin cycling may function as a quality-control mechanism to isolate damaged or depolarized mitochondria, which are less able to re-fuse with other healthy organelles $^{31}$, for degradation by autophagy, again consistent with a homeostatic role for this mechanism.

Constitutive actin cycling may be efficiently tuned to maintain mean mitochondrial size, complexity and number over each $14 \mathrm{~min}$ cycle, while also maintaining heterogeneity in the morphology of individual mitochondria. During mitosis, cells in prophase and metaphase were found to have both more fragmented mitochondria and actin around their mitochondria ${ }^{13}$. Thus, actin cycling may be upregulated in mitotic cells whose mitochondrial network must efficiently fragment in preparation for segregation into daughter cells ${ }^{32-34}$. Actin cycling may also regulate mitochondrial morphology in postmitotic cells such as neurons, which require precise regulation of mitochondrial dynamics and quality control for their viability ${ }^{35,36}$.

In summary, we propose a model in which mitochondrial network morphology is not regulated by a dynamic equilibrium between fission and fusion at the level of the individual organelle, but rather at a network level by the actin cytoskeleton. Mitochondria within the cell constantly grow and fuse, generating an increasingly branched and interconnected network. This steady growth is counterbalanced by intervals of fast and spatially restricted actin-dependent fission events, primarily at ER-mitochondrial contact sites. Although we focused primarily on the HeLa model system, we note similar cycles of actin assembly/disassembly regulating mitochondrial fission and fusion in another cell line, as well as in primary human cells, suggesting that actin-dependent cyclic mitochondrial fission may be a conserved homeostatic mechanism that functions to regulate the cellular mitochondrial network.

\section{Methods}

Reagents. Constructs used include: EGFP-actin (Clontech), LifeAct-EGFP, F-tractin-EGFP (Addgene), mCherry-LifeAct (Dominguez Lab, University of Pennsylvania), Mito-DsRed2 (gift from T. Schwarz, Harvard Medical School, Boston, MA) recloned into pSBFP2-C1 (Addgene) and SNAP-Tag (NEB), pKillerRed-dMito (Evrogen), Drp1 (Addgene) recloned into pEGFP (Clontech), Drp1-K38A (Addgene), DsRed2-ER (gift from A. Akhmanova, Utrecht University, Utrecht, The Netherlands).

Cell culture and transfections. HeLa-M cells (A. Peden, Cambridge Institute for Medical Research, Cambridge, UK) and COS-7 cells (ATCC) were cultured in DMEM medium (10-027-CV, Corning) supplemented with $10 \%$ fetal bovine serum (vol/vol) and $1 \%$ glutamax, and maintained at $37^{\circ} \mathrm{C}$ in a $5 \% \mathrm{CO}_{2}$ incubator. Normal human epidermal keratinocytes were isolated from de-identified discarded neonatal human foreskin, which was incubated for $12 \mathrm{~h}$ at $4 \mathrm{C}$ in $2.4 \mathrm{U} \mathrm{ml}^{-1}$ dispase $\mathrm{II}^{37}$. Sterile forceps were used to separate the underlying dermis.

The epidermal sheet was transferred to a $60 \mathrm{~mm}$ tissue culture plate and incubated in $0.25 \%$ Trypsin for $10 \mathrm{~min}$ at $37 \mathrm{C}$, then neutralized with $1 \mathrm{ml}$ fetal bovine serum. Sterile forceps were used to scrape the epidermal sheet against the dish to dissociate cells. The suspension was passed through a $40 \mu \mathrm{m}$ strainer, then centrifuged at $200 \mathrm{~g}$ for $5 \mathrm{~min}$. The cell pellet was re-suspended in $5 \mathrm{ml}$ medium M154 
(Invitrogen) containing $0.07 \mathrm{mM}$ calcium. Normal human epidermal keratinocytes were passaged up to six times. All cells were seeded in $35 \mathrm{~mm}$ glass-bottom dishes and transfected $\sim 24 \mathrm{~h}$ before imaging using FuGENE 6 (Promega). Cells were treated with $2 \mu \mathrm{M}$ Latrunculin B for $30 \mathrm{~min}$ (BML-T110-0001, Enzo), $25 \mu \mathrm{M}$ SMIFH2 for $1 \mathrm{~h}$ (S4826, Sigma), $84 \mu \mathrm{M}$ CK-666 for $1 \mathrm{~h}$ (SML0006, Sigma) or $50 \mu \mathrm{M}$ Mdivi-1 for 16 h (M0199, Sigma) before imaging. CCCP (Sigma-Aldrich) was given at $20 \mu \mathrm{M}$. Cells were incubated in $30 \mathrm{nM}$ TMRE in complete DMEM (T-669, Molecular Probes) for $15 \mathrm{~min}$ and washed $2 \times$ in DMEM before live-cell imaging. Cells expressing Mito-SNAP were incubated with $2.5 \mu \mathrm{M}$ SNAP-cell $647-\mathrm{SiR}$ (S9102S, NEB) for $30 \mathrm{~min}$ and washed $2 \times$ before imaging.

Live-cell imaging and analysis. All images were acquired on a spinning-disk confocal (UltraVIEW VoX; Perkin Elmer) on a Nikon Eclipse Ti microscope using an Apochromat $\times 1001.49$ numerical aperture oil-immersion objective (Nikon) in a temperature-controlled chamber $\left(37^{\circ} \mathrm{C}\right)$. Digital images were acquired with an EM charge-coupled device camera (C9100; Hamamatsu Photonics) using Volocity software (PerkinElmer) at 1 frame every $1-30$ s or 1 frame per minute. Specifically, after the plates are mounted on the microscope, we selected the first observed cell with identifiable actin recruitment to mitochondria. The cell was imaged for no longer than 15-20 min with $<10 \%$ laser power and then immediately a second cell was imaged in a separate area of the plate. No more than four cells were imaged from a single plate. Cells were excluded from analysis if they displayed signs of phototoxicity such as blebbing or vacuolization. All experiments were performed on at least three independent occasions. Time-lapse maximum intensity projection movies were created by acquiring nine $z$-slices separated by $0.5 \mu \mathrm{m}$ step size every $30 \mathrm{~s}$ for $15-20 \mathrm{~min}$. To induce ROS production, we photobleached a region or the entire HeLa cell expressing pKillerRed-dMito with a 561-nm laser at $100 \%$ for 100 iterations.

Images of mitochondria (both confocal slices and maximum intensity projections) were segmented using the pixel classification feature of the interactive learning and segmentation toolkit (Ilastik 1.1.5) and analysed by Fiji (NIH). Segmentation of TOM20 labelled mitochondria was carried out using Volocity (PerkinElmer). Automated image analysis was manually verified by comparing the segmentation mask to the original image files. Measures of mitochondrial area, number, circularity and fractional occupancy were generated using the 'analyse particles' function in Fiji (NIH) with a minimum area of $0.25 \mu \mathrm{m}^{2}$. Measures of mitochondrial length, junctions (voxels with three or more neighbors) and branches (slab segments connecting end points to either junctions or other endpoints) were determined using the 'skeletonize' and 'analyse skeleton' plugins in Fiji (NIH). Analyses were carried out on either whole cells or square $\sim 100 \mu \mathrm{m}^{2}$ ROIs around subpopulations of actin-positive mitochondria.

For actin recruitment analyses in Figs 4 and 8 , time 0 is defined as 1 min before the first observable actin recruitment to $>25 \%$ of mitochondria within the ROI. For actin disassembly analyses in Figs 5 and 8 , time 4 is defined as $1 \mathrm{~min}$ subsequent to the disassembly of actin from $>75 \%$ of mitochondria within the ROI. For Fig. 7, segmentation of mitochondria from both control and Drp1-K38A cells was carried out on maximum intensity projections and mitochondrial length and number was normalized per cell. All other analyses were performed on single plane images. Line scans were generated using ImageJ $(\mathrm{NIH})$ and normalized per fluorescent tag. Three-dimensional renderings were generated using Volocity (PerkinElmer). All images were assembled using Fiji (NIH) and Illustrator (Adobe). Statistics and graphing were performed using Prism (GraphPad) software. Comparisons of two data sets were performed using unpaired two-tailed Student's $t$-test for normally distributed data sets and Mann-Whitney test for non-normally distributed data sets. Comparisons for multiple data sets were performed using one-way analysis of variance with Tukey's post-hoc test for normally distributed data sets and Kruskal-Wallis test with Dunn's multiple comparison test for non-normally distributed data sets.

Immunofluorescence. HeLa cells seeded on $35 \mathrm{~mm}$ glass-bottom dishes were fixed in $3.7 \%$ paraformaldehyde at $37^{\circ} \mathrm{C}$ for $12 \mathrm{~min}$, washed in PBS $\times 3$ and permeabilized in $0.2 \%$ Triton X-100 in PBS for no longer than $5 \mathrm{~min}$ at room temperature. Cells were again washed in PBS $\times 3$ and blocked in $0.5 \%$ BSA in PBS for $10 \mathrm{~min}$ at room temperature. Cells were then incubated for $30 \mathrm{~min}$ with Phalloidin-488 (1:200; A12379, ThermoFisher), anti-Tom20 rabbit polyclonal antibody (1:50; sc-11415, Santa Cruz) and/or anti-DRP1 mouse monoclonal antibody (1:100; ab56788, Abcam) in PBS with $0.5 \%$ BSA. Cells were then washed in PBS $\times 3$ and incubated with either AlexaFluor goat-anti-rabbit-594 (1:300; A-11037, ThermoFisher) or AlexaFluor goat-anti-rabbit 555 (1:300; A21429, ThermoFisher), AlexaFluor goat-anti-mouse-647 (1:300; A21052, ThermoFisher) and Hoechst dye (62249; ThermoFisher). Cells were washed in PBS $\times 3$ and imaged.

Data availability. The data that support the findings of this study are available from the corresponding author upon request.

\section{References}

1. Mishra, P. \& Chan, D. C. Mitochondrial dynamics and inheritance during cell division, development and disease. Nat. Rev. Mol. Cell Biol. 15, 634-646 (2014).
2. Friedman, J. R. \& Nunnari, J. Mitochondrial form and function. Nature 505, 335-343 (2014).

3. Youle, R. J. \& van der Bliek, A. M. Mitochondrial fission, fusion, and stress. Science 337, 1062-1065 (2012).

4. Chen, H. \& Chan, D. C. Physiological functions of mitochondrial fusion. Ann N. Y. Acad. Sci. 1201, 21-25 (2010).

5. Santel, A. \& Fuller, M. T. Control of mitochondrial morphology by a human mitofusin. J. Cell. Sci. 114, 867-874 (2001).

6. Cipolat, S., Martins de Brito, O., Dal Zilio, B. \& Scorrano, L. OPA1 requires mitofusin 1 to promote mitochondrial fusion. Proc. Natl Acad. Sci. USA 101, 15927-15932 (2004)

7. Labrousse, A. M., Zappaterra, M. D., Rube, D. A. \& van der Bliek, A. M. C. elegans dynamin-related protein DRP-1 controls severing of the mitochondrial outer membrane. Mol. Cell 4, 815-826 (1999).

8. Losón, O. C., Song, Z., Chen, H. \& Chan, D. C. Fis1, Mff, MiD49, and MiD51 mediate Drp1 recruitment in mitochondrial fission. Mol. Biol. Cell 24, 659-667 (2013).

9. Friedman, J. R. et al. ER tubules mark sites of mitochondrial division. Science 334, 358-362 (2011).

10. Korobova, F., Ramabhadran, V. \& Higgs, H. N. An actin-dependent step in mitochondrial fission mediated by the ER-associated formin INF2. Science 339, 464-467 (2013).

11. Korobova, F., Gauvin, T. J. \& Higgs, H. N. A role for myosin II in mammalian mitochondrial fission. Curr. Biol. 24, 409-414 (2014).

12. Manor, U. et al. A mitochondria-anchored isoform of the actin-nucleating spire protein regulates mitochondrial division. Elife 4, e08828 (2015).

13. Li, S. et al. Transient assembly of F-actin on the outer mitochondrial membrane contributes to mitochondrial fission. J. Cell Biol. 208, 109-123 (2015).

14. Ji, W. K., Hatch, A. L., Merrill, R. A., Strack, S. \& Higgs, H. N. Actin filaments target the oligomeric maturation of the dynamin GTPase Drp1 to mitochondrial fission sites. Elife 4, el1553 (2015).

15. Riedl, J. et al. Lifeact: a versatile marker to visualize F-actin. Nat. Methods 5, 605-607 (2008).

16. Belin, B. J., Goins, L. M. \& Mullins, R. D. Comparative analysis of tools for live cell imaging of actin network architecture. Bioarchitecture 4, 189-202 (2014).

17. Verburg, J. \& Hollenbeck, P. J. Mitochondrial membrane potential in axons increases with local nerve growth factor or semaphorin signaling. J. Neurosci. 28, 8306-8315 (2008).

18. Bulina, M. E. et al. A genetically encoded photosensitizer. Nat. Biotechnol. 24 , 95-99 (2006).

19. Smirnova, E., Shurland, D. L., Ryazantsev, S. N. \& van der Bliek, A. M. A human dynamin-related protein controls the distribution of mitochondria. J. Cell Biol. 143, 351-358 (1998).

20. Cassidy-Stone, A. et al. Chemical inhibition of the mitochondrial division dynamin reveals its role in Bax/Bak-dependent mitochondrial outer membrane permeabilization. Dev. Cell 14, 193-204 (2008).

21. English, A. R. \& Voeltz, G. K. Endoplasmic reticulum structure and interconnections with other organelles. Cold Spring Harb. Perspect. Biol. 5, a013227 (2013).

22. Tee, Y. H. et al. Cellular chirality arising from the self-organization of the actin cytoskeleton. Nat. Cell Biol. 17, 445-457 (2015).

23. Harder, Z., Zunino, R. \& McBride, H. Sumol conjugates mitochondrial substrates and participates in mitochondrial fission. Curr. Biol. 14, 340-345 (2004).

24. Chang, C. R. \& Blackstone, C. Dynamic regulation of mitochondrial fission through modification of the dynamin-related protein Drp1. Ann. N. Y. Acad. Sci. 1201, 34-39 (2010).

25. Cagalinec, M. et al. Principles of the mitochondrial fusion and fission cycle in neurons. J. Cell. Sci. 126, 2187-2197 (2013).

26. Boldogh, I. R. et al. Arp2/3 complex and actin dynamics are required for actin-based mitochondrial motility in yeast. Proc. Natl Acad. Sci. USA 98, 3162-3167 (2001).

27. Parone, P. A. et al. Preventing mitochondrial fission impairs mitochondrial function and leads to loss of mitochondrial DNA. PLoS ONE 3, e3257 (2008)

28. Legros, F., Malka, F., Frachon, P., Lombès, A. \& Rojo, M. Organization and dynamics of human mitochondrial DNA. J. Cell. Sci. 117, 2653-2662 (2004).

29. Zajac, A. L., Goldman, Y. E., Holzbaur, E. \& Ostap, E. M. Local cytoskeletal and organelle interactions impact molecular-motor-driven early endosomal trafficking. Curr. Biol. 23, 1173-1180 (2013).

30. Lee, S. et al. Mitochondrial fission and fusion mediators, hFis1 and OPA1, modulate cellular senescence. J. Biol. Chem. 282, 22977-22983 (2007).

31. Twig, G. et al. Fission and selective fusion govern mitochondrial segregation and elimination by autophagy. EMBO J. 27, 433-446 (2008).

32. Taguchi, N., Ishihara, N., Jofuku, A., Oka, T. \& Mihara, K. Mitotic phosphorylation of dynamin-related GTPase Drpl participates in mitochondrial fission. J. Biol. Chem. 282, 11521-11529 (2007).

33. Zunino, R., Braschi, E., Xu, L. \& McBride, H. M. Translocation of SenP5 from the nucleoli to the mitochondria modulates DRP1-dependent fission during mitosis. J. Biol. Chem. 284, 17783-17795 (2009). 
34. Kashatus, D. F. et al. RALA and RALBP1 regulate mitochondrial fission at mitosis. Nat. Cell Biol. 13, 1108-1115 (2011).

35. Chen, H. \& Chan, D. C. Critical dependence of neurons on mitochondrial dynamics. Curr. Opin. Cell Biol. 18, 453-459 (2006).

36. Maday, S. \& Holzbaur, E. L. Autophagosome biogenesis in primary neurons follows an ordered and spatially regulated pathway. Dev. Cell 30, 71-85 (2014).

37. Simpson, C. L., Kojima, S. \& Getsios, S. RNA interference in keratinocytes and an organotypic model of human epidermis. Methods Mol. Biol. 585, 127-146 (2010).

\section{Acknowledgements}

We thank Mariko Tokito for technical assistance, and Pedro Guedes-Dias and David Kast for helpful advice. This work was supported by the National Institute of Health Grant P01 GM087253 (E.L.F.H.). C.L.S. was supported by NIH/NIAMS grant T32 AR007465.

\section{Author contributions}

A.S.M. co-designed the study, performed experiments, analysed the data and wrote the manuscript. Y.C.W. co-designed the study, performed experiments, analysed the data and wrote the manuscript. C.L.S. performed experiments and edited the manuscript. E.L.F.H. co-designed the study and wrote the manuscript.

\section{Additional information}

Supplementary Information accompanies this paper at http://www.nature.com/ naturecommunications

Competing financial interests: The authors declare no competing financial interests.

Reprints and permission information is available online at http://npg.nature.com/ reprintsandpermissions/

How to cite this article: Moore, A. S. et al. Dynamic actin cycling through mitochondrial subpopulations locally regulates the fission-fusion balance within mitochondrial networks. Nat. Commun. 7:12886 doi: 10.1038/ncomms12886 (2016).

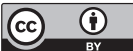

This work is licensed under a Creative Commons Attribution 4.0 International License. The images or other third party material in this article are included in the article's Creative Commons license, unless indicated otherwise in the credit line; if the material is not included under the Creative Commons license, users will need to obtain permission from the license holder to reproduce the material. To view a copy of this license, visit http://creativecommons.org/licenses/by/4.0/

(C) The Author(s) 2016 